\title{
Noninvasive ventilation: has Pandora's box been opened?
}

This article was published in the following Dove Press journal:

International Journal of COPD

26 February 2010

Number of times this article has been viewed

\section{Ari Manuel' \\ Richard EK Russell ${ }^{2}$ \\ Quentin Jones ${ }^{3}$}

'Department of Respiratory Medicine, High Wycombe Hospital, Bucks, UK;

${ }^{2}$ Airway Disease Section, National Heart and Lung Institute, Imperial College, London, UK; ${ }^{3}$ Specialist Registrar, Department of Respiratory Medicine, Churchill Hospital, Oxford

Correspondence: Dr Ari Manuel Department of Respiratory Medicine, High Wycombe Hospital, Queen Alexandra Road, High Wycombe, Bucks, HPII 2TT, UK Email arimanuel1979@yahoo.co.uk
Exacerbations of COPD are the largest single cause of hospital admission with respiratory disease, and are frequently associated with impaired gas exchange and mortality rates of up to $14 \% .{ }^{1}$ Acute hypercapnic respiratory failure leads to admissions to intensive care units with a mortality rate of $59 \%$ at one year. ${ }^{2}$

Noninvasive ventilation (NIV) is a well established and validated therapy for acidotic hypercapnia respiratory failure in COPD, ${ }^{1}$ a leading cause of global mortality and morbidity. The use of NIV in patients with acute type II or chronic respiratory failure has increased over the past 10 years.

A Cochrane Systematic Review determined the efficacy of NIV in the management of patients with respiratory failure due to an acute exacerbation of COPD. NIV resulted in decreased mortality, decreased need for intubation, and a reduction in treatment failure. ${ }^{1}$ However a Royal College of Physicians/British Thoracic Society (RCP/BTS) audit failed to provide evidence that NIV was effective in reducing mortality. The reason for their finding is unclear. ${ }^{3}$

There is little convincing evidence for the use of NIV in severe, but stable COPD. In many cases, patients with severe chronic COPD may not tolerate long-term NIV. However, NIV has been accepted as the convention for ventilation support for patients who have developed progressive type II respiratory failure. ${ }^{4}$

What is less clear, however, is the quality of how NIV is delivered to patients in hospitals in the UK.

The RCP/BTS audit of NIV use in 233 hospitals showed NIV was available in all but 11 hospitals, but only $31 \%$ of patients who were admitted with hypercapnic acidotic exacerbations of COPD $(\mathrm{pH}<7.35)$ received NIV. ${ }^{3}$

A recent update of the guidelines of NIV has reinforced much of current practice but thrown up several interesting questions. ${ }^{5}$

Data from the recent BTS National COPD audit (NCROP) demonstrated large gaps in training of staff, provision of written guidelines and the ability to audit practice. $^{6}$

Any patient on NIV is classified as receiving Critical Level 2 care, defined as "Patients requiring more detailed observation or intervention including support for a single failed organ system". This suggests NIV should be administered in an intensive care unit (ICU) or high dependency unit (HDU) setting, but it has been widely recognised that NIV can be successfully used outside the ICU or HDU. 
In the UK previous studies have shown that $40 \%$ of NIV is performed on general medical/respiratory wards, $12 \%$ by HDUs and $13 \%$ by ICU. ${ }^{2}$

We conducted a prospective review of NIV practice at our hospital in order to compare local practice against guideline stated best practice. In this review, conducted over one year, we noted that $64 \%$ of patients were started on NIV in the accident and emergency department. The new guidelines suggest that a dedicated area of high-dependency care is needed with appropriately trained staff. Certainly most emergency departments have this, but efforts need to be made to ensure training is adequate and that staff keeps up to date.

Concerns have been raised about the use of NIV in situations other than hypercapnic respiratory failure in the context of COPD. Technological advances have made the technique relatively simple and thus we are concerned that NIV is being inappropriately used, most often in the wrong indication. This may result in serious negative clinical consequences. In our review of local practice, we found $55 \%$ of patients were receiving NIV for either heart failure or pneumonia, neither of which are indications for use of NIV in the BTS guidelines. $^{5}$

In the RCP survey, of the 1095 patients who were not commenced on NIV despite fulfilling criteria, the reasons for not starting NIV included "inappropriate" in 321, "no facilities" in 48, "patient refused" in 13, "treatment failed" in 3 , with no reason offered in $710 .^{3}$

A review of NIV use in patients with acute cardiogenic pulmonary edema showed a significant reduction in the mortality rate by nearly $45 \%$ compared with conventional therapy. There was also a significant decrease in the "need to intubate" rate. However, continuous positive airway pressure or bilevel positive airway pressure showed no differences in intubation or mortality rates in the analysis of studies comparing the two techniques. ${ }^{7}$

The new guidelines recommend that efforts must be now made to comment on gaining the patient's consent and document the reasoning for the use of NIV. From a patient's perspective, more compliance would be gained if the patient felt they had some control of the process and if a variety of interfaces were tried. But in a busy emergency department, these are often not feasible. We found poor documentation on patient's consent to treatment and also almost one in seven had no documentation of why NIV was instituted.

The new guidelines also state that at least a second year speciality trainee (ST2) must make the decision on the commencement of NIV, but we found that in $25 \%$ of cases this was not the case. This is especially difficult in most emergency units as they are often staffed by a mixture of nonspecialist junior and middle-grade trainees.

NIV is a very well validated and successful treatment when used for the right indication, with the correctly trained staff and with motivated patients. At present we believe that there are problems common to many emergency and respiratory departments which urgently need addressing.

Solutions need to be designed and implemented by local teams. They might include a dedicated NIV team, able to provide 24/7 care. This is, however, only feasible in large units with many trained staff. For smaller units lead staff should be trained regularly to maintain knowledge and competencies for this important intervention, which is most often utilized in the period termed "out-of hours".

\section{References}

1. Ram FS, et al. Non-invasive positive pressure ventilation for treatment of respiratory failure due to exacerbations of chronic obstructive pulmonary disease. Cochrane Database Syst Rev. 2004;(1): CD004104.

2. Gorini M, et al. Non-invasive negative and positive pressure ventilation in the treatment of acute on chronic respiratory failure. Intensive Care Med. 2004;30:875-881.

3. Kaul S, Couts I, Lowe D, et al. Survey of NIV in clinical management of acute COPD in 233 UK hospitals. Thorax. 2007;62:S111-A120.

4. Meecham Jones DJ, Wedzicha JA. Comparison of pressure and volume preset nasal ventilator systems in stable chronic respiratory failure. Eur Respir J. 1993;6:1060-1064.

5. Non-invasive ventilation in chronic obstructive pulmonary disorder: management of acute type 2 respiratory failure. RCP/BTS Concise Guideline, October 2008.

6. National COPD Resources and Outcomes Project [NCROP] http://www. britthoracic.org.uk/ClinicalInformation/COPD/NationalCOPDResourcesandOutcomesProject/tabid/362/Default.aspx.

7. Masip J, Roque M, Sanchez B. Noninvasive ventilation in acute cardiogenic pulmonary edema: systematic review and meta-analysis. JAMA. $2005 ; 294: 3124-3130$.
International Journal of COPD

\section{Publish your work in this journal}

The International Journal of COPD is an international, peer-reviewed journal of therapeutics and pharmacology focusing on concise rapid reporting of clinical studies and reviews in COPD. Special focus is given to the pathophysiological processes underlying the disease, intervention programs, patient focused education, and self management protocols.

\section{Dovepress}

This journal is indexed on PubMed Central, MedLine and CAS. The manuscript management system is completely online and includes a very quick and fair peer-review system, which is all easy to use. Visit $\mathrm{http}: / /$ www.dovepress.com/testimonials.php to read real quotes from published authors. 